\title{
Focused Proteomics Revealed a Novel Rho-kinase Signaling Pathway in the Heart
}

\author{
Yoshimitsu Yura ${ }^{1,2}$, Mutsuki Amano ${ }^{1}$, Mikito Takefuji ${ }^{2}$, Tomohiro Bando ${ }^{1}, K_{0 u}$ Suzuki ${ }^{1}$, \\ Katsuhiro Kato ${ }^{1,2}$, Tomonari Hamaguchi ${ }^{1}$, Md. Hasanuzzaman Shohag ${ }^{1}$, Tetsuya Takano, \\ Yasuhiro Funahashi ${ }^{1}$, Shinichi Nakamuta ${ }^{1}$, Keisuke Kuroda ${ }^{1}$, Tomoki Nishioka ${ }^{1}$, Toyoaki Murohara ${ }^{2}$, \\ and Kozo Kaibuchi ${ }^{1 *}$ \\ ${ }^{1}$ Department of Cell Pharmacology, Graduate School of Medicine, Nagoya University, 65 Tsurumai, Showa-ku, \\ Nagoya 466-8550, Japan, ${ }^{2}$ Department of Cardiology, Graduate School of Medicine, Nagoya University, 65 \\ Tsurumai, Showa-ku, Nagoya 466-8550, Japan
}

\begin{abstract}
Protein phosphorylation plays an important role in the physiological regulation of cardiac function. Myocardial contraction and pathogenesis of cardiac diseases have been reported to be associated with adaptive or maladaptive protein phosphorylation; however, phosphorylation signaling in the heart is not fully elucidated. We recently developed a novel kinase-interacting substrate screening (KISS) method for exhaustive screening of protein kinase substrates, using mass spectrometry and affinity chromatography. First, we examined protein phosphorylation by extracellular signal-regulated kinase (ERK) and protein kinase A (PKA), which has been relatively well studied in cardiomyocytes. The KISS method showed that ERK and PKA mediated the phosphorylation of known cardiac-substrates of each kinase such as Rps6ka1 and cTnI, respectively. Using this method, we found about 330 proteins as Rho-kinase-mediated substrates, whose substrate in cardiomyocytes is unknown. Among them, CARP/Ankrd1, a muscle ankyrin repeat protein, was confirmed as a novel Rho-kinasemediated substrate. We also found that non-phosphorylatable form of CARP repressed cardiac hypertrophyrelated gene Myosin light chain-2v (MLC-2v) promoter activity, and decreased cell size of heart derived H9c2 myoblasts more efficiently than wild type-CARP. Thus, focused proteomics enable us to reveal a novel signaling pathway in the heart.
\end{abstract}

Key words: CARP, kinase, mass spectrometry, phosphorylation, ROCK

\section{Introduction}

Protein phosphorylation is a major and essential posttranslational modification that mediates intracellular signal transduction in cardiomyocytes. Adaptive phosphorylation mediates the contraction and relaxation of cardiomyocytes to regulate cardiac pump function (Bers, 2002). Conversely, maladaptive phosphorylation causes cardiac diseases such as cardiac hypertrophy and heart failure (Tilley, 2011). Thus, analyzing the phosphorylation process could aid in better understanding of the physiological and pathological functions of the heart.

\footnotetext{
*To whom correspondence should be addressed: Kozo Kaibuchi, Department of Cell Pharmacology, Graduate School of Medicine, Nagoya University, 65 Tsurumai, Showa-ku, Nagoya 466-8550, Japan.

E-mail: kaibuchi@med.nagoya-u.ac.jp

Abbreviations: CARP, Cardiac ankyrin repeat protein; cTnI, Cardiac troponin I; ERK, Extracellular signal-regulated kinase; KISS, Kinaseinteracting substrate screening; LC-MS/MS, Liquid chromatography tandem mass spectrometry; MYPT1, Myosin phosphatase target subunit 1; PKA, Protein kinase A.
}

Some cardiomyocyte-specific phosphorylated proteins, such as phospholamban, L-type $\mathrm{Ca}^{2+}$ channels, and cardiac troponin I, are examined by traditional ${ }^{32} \mathrm{P}$-labeling methods that involve incorporation of ${ }^{32} \mathrm{P}$ into cellular proteins by the treatment with radiolabeled ATP (Yan et al., 1998). The radioactive method is useful for individual examination of well-phosphorylated proteins; however, the exhaustive screening of finely phosphorylated proteins is difficult. Alternatively, some of the phosphorylation processes examined in cardiomyocytes were originally detected in noncardiac cells, such as hepatocytes and COS- 1 cells derived from the kidney. In fact, very few cardiomyocyte-specific phosphoproteins have been detected in the last 20 years by the traditional methods.

Recently, liquid chromatography tandem mass spectrometry (LC-MS/MS)-based phosphoproteomics has become increasingly popular for identification of global phosphopeptides. Gygi et al. reported more than 7,000 phosphorylation sites in mouse heart proteins (Huttlin et al., 2010), and Olsen et al. reported beta-adrenaline-mediated phosphory- 
lation sites in murine hearts (Lundby et al., 2013), suggesting that a large number of proteins are phosphorylated in the heart. Such conventional LC-MS/MS-based methods could identify the phosphorylated proteins and their phosphorylation sites; however, it is still unclear which kinases phosphorylate the respective sites. Therefore, the phosphorylation signaling pathway in the heart is largely unknown.

In this study, we performed exhaustive substrate screening of extracellular signal-regulated kinase (ERK), protein kinase A (PKA), and Rho-kinase/ROCK using the Kinaseinteracting substrate screening (KISS) method, which we recently developed (Amano et al., 2015, 2010b), because these kinases have significant roles in the heart. Although several substrates of ERK and PKA in cardiomyocytes have been reported, little is known about cardiac specific substrate of Rho-kinase, despite its significant role in the heart (Hartmann et al., 2015; Shimokawa and Satoh, 2015). In the ERK and PKA experiments, we detected 242 and 382 substrates, respectively, including major known substrates, suggesting the efficacy of the KISS method as an initial substrate screening in the heart. In the Rho-kinase experiment, we also detected 329 potential substrates. Among them, CARP/Ankrd1, a muscle ankyrin repeat protein, was confirmed as a novel substrate of Rho-kinase. We show that Rho-kinase directly phosphorylates CARP and that phosphorylation of CARP by Rho-kinase appears to attenuate its function as a transcriptional regulator.

\section{Materials and Methods}

\section{Material and chemicals}

Anti-phospho-PKA substrate $\left(\mathrm{RRXS}^{*} / \mathrm{T}^{*}\right)$ rabbit monoclonal antibody, anti-( $\left.\mathrm{T}^{*} \mathrm{P}\right)$ rabbit monoclonal antibody, anti-Troponin I rabbit polyclonal antibody, anti-Rps6kal rabbit monoclonal antibody, anti-phospho-Rps6ka1 (Ser380) rabbit monoclonal antibody, and anti-Myc rabbit monoclonal antibody were purchased from Cell Signalling Technology (Massachusetts, USA). AntiCardiac Troponin I (phospho T143) rabbit polyclonal antibody was purchased from Abcam (Cambridge, United Kingdom). AntiCARP rabbit polyclonal antibody was purchased from Santa Cruz (Dallas, TX, USA). Anti-GFP mouse monoclonal antibody was purchased from Roche (Basel, Switzerland). Anti- $\alpha$-Actinin (Sarcomeric) mouse monoclonal antibody was purchased from SigmaAldrich (St Louis, MO, USA). GST-Rho-kinase-cat (bovine, residues 6-553) was expressed in Sf9 cells using a baculovirus system and purified using glutathione Sepharose beads (Amano et al., 1999). GST-PKACA and GST-MAPK1/Erk2 were provided by Y. Kirii and K. Yoshino (Carna Biosciences, Kobe, Japan). The cDNA encoding human CARP was purchased from Kazusa DNA Research Institute (Chiba, Japan). cDNA encoding mouse 14-3-3 was cloned from a human cDNA library. cDNAs encoding fulllength or fragments of CARP and 14-3-3 were cloned into pEGFP (Takara Bio. Inc., Japan), pEF-BOS-GST, and pGEX (GE Health- care UK Ltd., England) vectors. p(MLC2v) $)_{4}$-Luci reporter plasmid was a kind gift from Dr. E. Villard (Duboscq-Bidot et al., 2009). pGL4-p53RE-Luci reporter plasmid and trypsin for mass spectrometry were purchased from Promega Co. (Madison, WI). Y-27632 was purchased from Wako Pure Chemical Industries (Osaka, Japan). FR180204 and KT5720 were purchased from Santa Cruz (Dallas, TX, USA). Cy2- and Cy3-conjugated secondary antibodies against mouse and rabbit immunoglobulin $\mathrm{G}$ were purchased from Jackson ImmunoResearch Laboratories (West Grove, PA, USA). H9c2 cardiomyoblast cells were obtained from the American Type Culture Collection (Manassas, VA, USA). Transfections were carried out using Lipofectamine 2000 reagent (Thermo Fisher Scientific Inc., Waltham, MA) according to the manufacturer's protocol. Other materials and chemicals were obtained from commercial sources.

\section{Preparation of the rat heart cytosolic fraction}

Preparation of the rat heart cytosolic fraction was described as previously (Kato et al., 2012). Briefly, the cytosolic proteins were extracted from the frozen heart powder, and then precipitated with ammonium sulfate at a concentration of $60 \%$. The proteins were resuspended in buffer A $(20 \mathrm{mmol} / \mathrm{L}$ Tris- $\mathrm{HCl}, \mathrm{pH} 7.5,1 \mathrm{mmol} / \mathrm{L}$ EDTA, $1 \mathrm{mmol} / \mathrm{L} \mathrm{DTT}$, and $5 \mathrm{mmol} / \mathrm{L} \mathrm{MgCl}_{2}$ ), and dialyzed three times against buffer $\mathrm{A}$.

\section{Substrate screening (KISS method)}

The procedures of KISS method were essentially same as described previously (Amano et al., 2015). Briefly, GST-fused kinase (500 pmol) was immobilized on the beads, and mixed with the rat heart cytosolic fraction ( $5 \mathrm{mg}$ of protein). After washing, the kinase-substrate complexes were incubated with or without ATP and $\mathrm{Mg}^{2+}$ at $30^{\circ} \mathrm{C}$, and subjected to tryptic digestion. The digested peptides were applied to a Titansphere Phos-TiO tip (GL Sciences, Tokyo, Japan) followed by a desalting SPE C-TIP (AMR Inc., Tokyo, Japan) to concentrate phosphopeptides. The peptides were analysed by a Q Exactive mass spectrometry (Thermo Fisher Scientific Inc., Waltham, MA, USA) and an HTC-PAL autosampler and a Paradigm MS4 HPLC System (Michrom BioResources Inc., CA, USA). MaxQuant software (version 1.3.0.5) and the complete proteome set of Rattus norvegicus in UniProt KB (2014_07) were used to generate a peak list (Cox and Mann, 2008). The phosphopeptides with ion intensity ratio (phosphorylated sample/nonphosphorylated sample) $>5$ were picked up as candidate substrates. All kinases were analyzed twice.

\section{Phosphorylation assay}

GST-tagged CARP-FL was produced in E. coli, and purified protein and Rho-kinase-cat domain were mixed to induce phosphorylation. pEF-BOS-GST-CARP-FL (1-319 aa), pEF-BOS-GSTCARP-N-term (1-122 aa), pEF-BOS-GST-CARP-C-term (120319 aa), and their Ala mutants (CARP-FL-AA, CARP-N-102A, CARP-C-314A) were transfected into COS7 cells, and the precipi- 
tated proteins were subjected to in vitro phosphorylation assays. The phosphorylation reactions were performed in $50 \mu \mathrm{l}$ of a reaction mixture $(25 \mathrm{mmol} / \mathrm{L}$ Tris- $\mathrm{HCl}, \mathrm{pH} 7.5,1 \mathrm{mmol} / \mathrm{L}$ EDTA, 1 $\mathrm{mmol} / \mathrm{L}$ EGTA, $1 \mathrm{mmol} / \mathrm{L}$ DTT, $5 \mathrm{mmol} / \mathrm{L} \mathrm{MgCl}_{2}$, and $50 \mu \mathrm{mol} / \mathrm{L}$ $\left[\gamma-{ }^{32} \mathrm{P}\right]$ ATP $\left.[10-20 \mathrm{GBq} / \mathrm{mmol}]\right)$, GST-Rho-kinase-cat $(0.03-0.3$ $\mu \mathrm{mol} / \mathrm{L}$ ), and substrates for 10 or $30 \mathrm{~min}$ at $30^{\circ} \mathrm{C}$. The phosphorylated proteins were separated by SDS-PAGE and GST-CARP was visualized using silver staining. Phosphorylated GST-CARP was imaged by autoradiography. The radiolabeled proteins were analysed by an image analyser (FLA9000; GE Healthcare UK Ltd., England).

\section{Affinity chromatography}

Affinity chromatography of GST-CARP was performed essentially as previously described (Amano et al., 1996). The adult rat heart cytosol fraction was incubated with $200 \mu \mathrm{l}$ of glutathione Sepharose beads on which 400 pmol of phosphorylated or nonphosphorylated GST-CARP was immobilized. After washing three times with $1 \mathrm{ml}$ of buffer B $(20 \mathrm{mmol} / \mathrm{L}$ Tris/HCl, $\mathrm{pH} 7.5,1$ mmol/L EDTA, $1 \mathrm{mmol} / \mathrm{L}$ DTT) and $1 \mathrm{ml}$ of buffer B containing $50 \mathrm{mmol} / \mathrm{L} \mathrm{NaCl}$, the bound proteins were subsequently eluted with $125 \mu \mathrm{l}$ of buffer B containing $1 \mathrm{~mol} / \mathrm{L} \mathrm{NaCl}$. The first eluate was subjected to LC-MS/MS analysis.

\section{In vitro binding assay}

The interaction of CARP with 14-3-3 was examined using an in vitro binding assay as previously described (Amano et al., 1996). Briefly, GFP-CARP-WT or -CARP-102A314A (CARP-AA) was transfected into COS7 cells, and the cells were treated with either DMSO or Y-27632 (Rho-kinase-specific inhibitor). These lysates were incubated with purified GST or GST-14-3-3 protein in lysis buffer $(20 \mathrm{mmol} / \mathrm{L}$ Tris- $\mathrm{HCl}$ at $\mathrm{pH} 7.5,1 \mathrm{mmol} / \mathrm{L}$ EDTA, 1 $\mathrm{mmol} / \mathrm{L}$ DTT, $150 \mathrm{mmol} / \mathrm{L} \mathrm{NaCl}, 20 \mathrm{mmol} / \mathrm{L} \beta$-glycerophosphate, $20 \mathrm{mmol} / \mathrm{L} \mathrm{NaF}, 1 \% \mathrm{NP}-40,0.1 \mathrm{mmol} / \mathrm{L}$ APMSF, $0.5 \mu \mathrm{g} / \mathrm{mL}$ aprotinin, and $2 \mu \mathrm{g} / \mathrm{mL}$ leupeptin) for $1 \mathrm{~h}$ at $4{ }^{\circ} \mathrm{C}$ and pulled down with glutathione Sepharose. Pulled-down samples were subjected to immunoblot using GFP antibody.

\section{Immunocytochemistry}

$\mathrm{H} 9 \mathrm{c} 2$ cells and COS7 cells were seeded on a 13-mm glass coverslip coated with poly-D-lysine at a density of $1.0 \times 10^{4}$ cells in DMEM supplemented with 10\% FBS and cultured overnight. GFP-14-3-3 $\zeta$-WT or -phospho-motif binding-deficient mutant (R56A/R60A) was transfected into H9c2 cells. Myc-CARP-WT or -AA was transfected with COS7 cells in combination with GFP or GFP-14-3-3. Twenty-four hours after transfection, cells were fixed with $3.7 \%$ formaldehyde for $10 \mathrm{~min}$ and washed with PBS at room temperature. Cells were incubated with anti-GFP, antiCARP, and anti-Myc antibodies for $60 \mathrm{~min}$ at room temperature. After washing, the samples were incubated with a Cy3- or Cy2conjugated secondary antibody and Hoechst. Fluorescence was examined using a laser confocal microscope (LSM780, Carl Zeiss, Obeokochem, Germany).

\section{Primary Isolation of Cardiomyocytes and Cell Culture}

Primary cardiomyocytes were isolated from rats at postnatal day $0-1$ using the Worthington neonatal cardiomyocyte isolation system (Worthington Biochemical Corp., City, Country) according to the manufacturer's instructions. A preplating step (for 2 hours) was included to reduce the number of fibroblasts in the cultures. The cells were then plated on 24-well dishes at a density of $3.0 \times 10^{4}$ cells with DMEM containing $10 \%$ FBS. The purity of myocytes in the culture was approximately $80 \%$, which was estimated by immunofluorescent staining with an anti-sarcomeric actinin antibody. These cells were used for experiments 24 hours after plating.

\section{Luciferase assay}

The Dual-Luciferase Reporter Assay System (Promega) was used to carry out transactivation experiments using a MLC-2v promoter and a 533 responsive promoter cloned upstream of the firefly luciferase reporter gene. Neonatal rat cardiomyocytes and HEK293 cells were grown for 24 hours and then transiently co-transfected using Lipofectamine 2000 with reporter vectors $\left(\mathrm{pGL}_{3}-(\mathrm{MLC}-2 \mathrm{v})_{4}\right.$ promoter or $\mathrm{pGL}_{4}-\mathrm{p} 53-\mathrm{RE}$ promoter in combination with the $\mathrm{pRL}$ TK promoter) and expression vectors for GFP-CARP-WT or CARP-AA. The cells were lysed in Passive Lysis Buffer 24 hours after transfection, and luciferase activity was measured using the Dual-Luciferase Assay System and a luminometer (Power Scan4, DS Pharma Biomedical, City, Country). Firefly luciferase activities were normalized to their corresponding renilla luciferase activities to correct for variations in transfection efficiency. The plotted results are representative of three independent experiments performed in sextuplicate.

\section{Measurement of cell size}

H9c2 cells were seeded on a 13-mm glass coverslip coated with poly-D-lysine at a density of $1.0 \times 10^{4}$ cells in DMEM supplemented with $10 \%$ FBS and were cultured overnight. GFP, GFPCARP-WT or -AA were transfected into H9c2 cells and medium was changed to DMEM. Twenty-four hours after transfection, cells were treated with $10 \%$ FBS for 24 hours before fixation in $3.7 \%$ formaldehyde. Fixed H9c2 cells were stained with anti-GFP and anti-actinin antibodies for $60 \mathrm{~min}$ at room temperature. After washing, the samples were incubated with a Cy3- or Cy2conjugated secondary antibody and Hoechst. Fluorescence was examined using a laser confocal microscope (LSM780, Carl Zeiss, Obeokochem, Germany). Individual H9c2 cell areas were measured using the ImageJ software. The mean values of 100 cell areas were measured for each vector. Results are representative of three independent experiments.

\section{Statistical analysis}

One-way analysis of variance (ANOVA) was performed after data were confirmed to satisfy the criteria of normal distribution and 
equal variance. If the overall ANOVA was significant, we performed a post hoc test, and the Tukey multiple comparison test was carried out.

\section{Results}

\section{KISS efficiently identified the substrates and phosphorylation sites of ERK and PKA in the heart}

We used affinity chromatography to isolate the substrates of protein kinases. Combining rat heart lysate and affinity beads coated with protein kinase facilitated the formation of the kinase-substrate complex (Fig. 1A). The proteins interacted with the kinase were incubated with or without ATP to induce phosphorylation. The phosphopeptides were submitted to LC-MS/MS analysis after trypsin digestion and concentration with a titanium oxide column. Previously, we found the potential substrates for Rho-kinase from rat brain lysate by this approach, and refer to this method as the kinase-interacting substrate screening (KISS) method (Amano et al., 2015).

As ERK and PKA have been well studied in cardiomyocytes, we initially used this method to detect ERK- or PKA-mediated substrates in the heart. To examine the phosphorylation in the kinase-substrate complex, the complexes were subjected to immunoblot before tryptic digestion using antibodies recognizing phospho-T-P and K/R-K/ R-X-phospho-S/T, which are favorable phosphorylated sequences by ERK and PKA, respectively. Immunoblot analyses with the corresponding motif antibodies showed a lot of bands in the presence of ATP and few bands in the absence of ATP (Fig. 1B). ERK inhibitor FR180204 or PKA inhibitor KT5720 treatment before adding ATP reduced the phosphorylation of substrates (Fig. 1C). These results suggest that ERK and PKA substantially phosphorylated the substrates interacted with them under the conditions. After confirmation of phosphorylation of ERK- or PKA-mediated substrate complexes, the complexes were examined by LC-MS/MS analysis, which showed 107 ERK-specific, 247 PKA-specific, and 135 common candidate substrates. Moreover, 551 and 881 localized phosphorylation sites were found in the ERK and PKA experiments, respectively (S1, 2, and 3 Tables). Of note, most of the phosphorylation sites in the common candidate substrates by ERK and PKA are distinct from each other. The majority of the candidate substrates were newly detected (Table I).

Table I. The NUmber of CANDidate substrates FOR ERK AND PKA DETERMINED BY KISS METHOD

\begin{tabular}{lccc}
\hline & $\begin{array}{c}\text { Detected in } \\
\text { the KISS method }\end{array}$ & $\begin{array}{c}\text { Known } \\
\text { substrates }\end{array}$ & $\begin{array}{c}\text { Newly detected } \\
\text { substrates }\end{array}$ \\
\hline ERK & 242 & 28 & 214 \\
PKA & 382 & 42 & 340 \\
\hline
\end{tabular}

These proteins included the well-known substrates such as Rps6ka1 and Mknk1 for ERK, and cTnI/Tnni3 and Mybpc3 for PKA. Consistent with the previous reports (Ubersax and Ferrell, 2007), sequence alignment of the predicted phosphorylation sites revealed that ERK and PKA preferentially phosphorylate $\mathrm{p}(\mathrm{S} / \mathrm{T}) \mathrm{P}$ and $(\mathrm{K} / \mathrm{R})(\mathrm{K} / \mathrm{R}) \mathrm{Xp}(\mathrm{S} / \mathrm{T})$ sequences, respectively (Fig. 2A). The phosphorylation sites detected in cTnI and Rps6kal by LC-MS/MS analysis were confirmed by immunoblot of the kinase-substrate complexes using phosphorylation site-specific antibodies (Fig. 2B). These results suggest that this method efficiently identifies kinase-mediated potential substrates and phosphorylation sites.

To address whether the phosphorylated proteins by ERK or PKA fell into clusters of interconnected proteins, we performed pathway analysis with our identified phosphoproteins as seeds. KEGG pathway analysis showed that the ERK-specific substrates are involved in not only Mitogenactivated protein kinases (MAPK) signaling but also mammalian target of rapamycin (mTOR) signaling pathway (Fig. 2C). In addition to cardiac muscle contraction-related proteins, the PKA-specific substrates are involved in the metabolic processes such as glycolysis/gluconeogenesis and insulin signaling (Fig. 2C). These results suggest that ERK and PKA are supposed to be involved in the wider range of functions than previously reported.

\section{Novel substrates of Rho-kinase in the heart}

Rho-kinase/ROCK, an effector of the small GTPase Rho, mediates the rearrangements of the actomyosin cytoskeleton in smooth muscles (Amano et al., 2010a). A series of translational research studies have demonstrated the important roles of Rho-kinase in the pathogenesis of coronary artery spasm (Hartmann et al., 2015; Shimokawa and Satoh, 2015). Although the function of Rho-kinase in the heart has been less studied compared with smooth muscle cells, there is growing evidence that the Rho and Rhokinase pathway plays an important role in the development of cardiac diseases such as cardiac hypertrophy and heart failure (Hartmann et al., 2015; Shimokawa and Satoh, 2015). It is necessary to examine the substrates of Rhokinase in order to elucidate its role in cardiac diseases.

We investigated the substrates phosphorylated by Rhokinase in the heart using the KISS method. The phosphorylation of substrates in the substrate-kinase complex was detected in the presence of ATP by immunoblot using antibody recognizing K/R-K/R-X-phospho-S/T (Fig. 3A), which was reduced by the treatment of Rho-kinase inhibitor Y-27632 (Fig. 3B). These results suggest that Rho-kinase substantially phosphorylated the substrates interacted with it. As a result of LC-MS/MS study, a large number of proteins including, cytoskeletal protein, sarcomere protein, kinase, regulators of small GTPases (GEF/GAP), and transcriptional regulator were detected (Fig. 3C, S4 and S5 


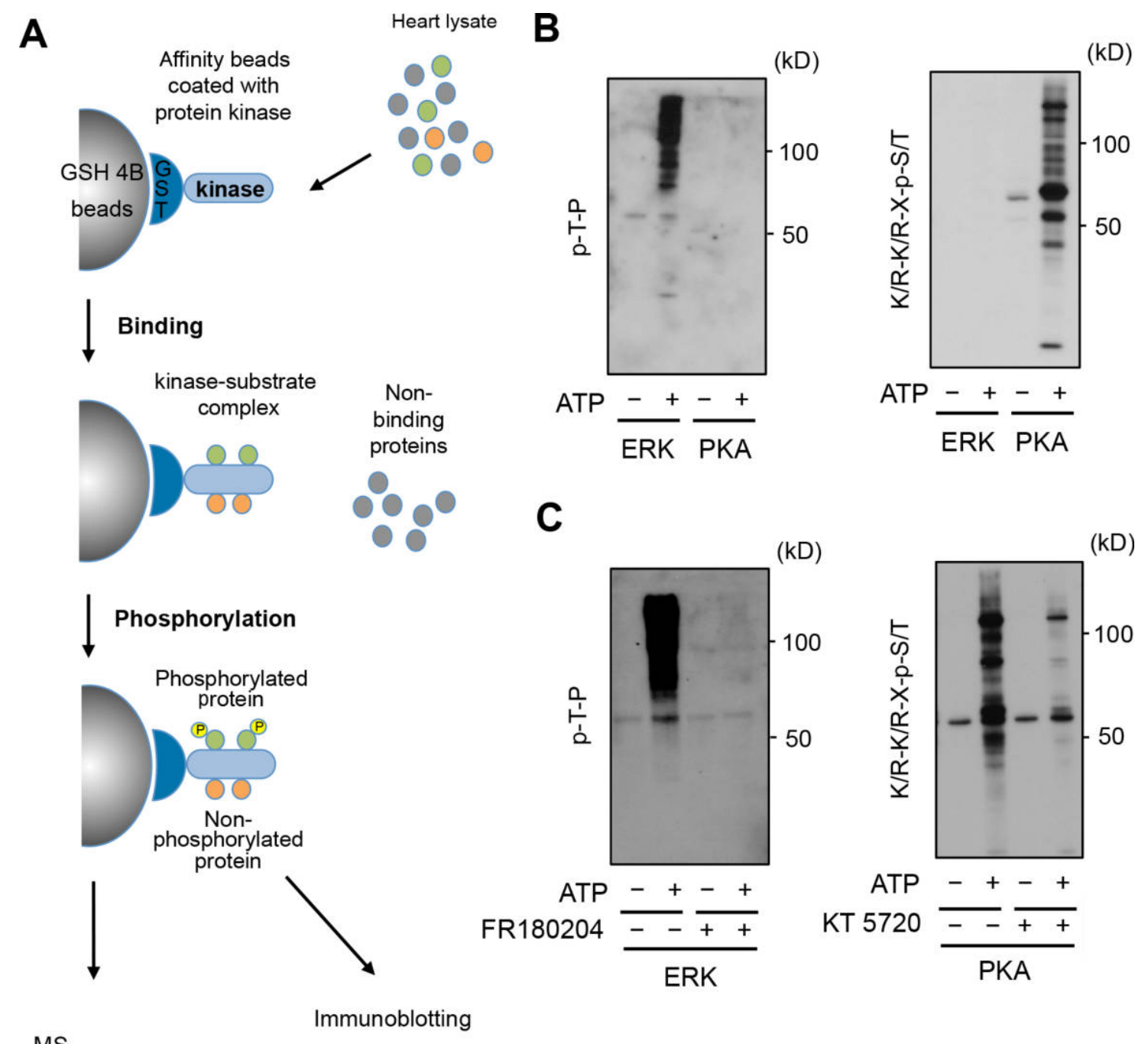

MS
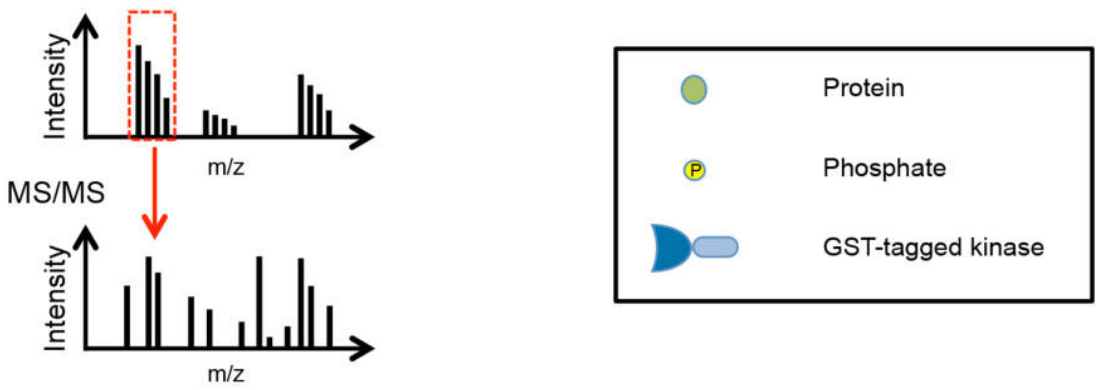

LC-MS/MS

Fig. 1. The Scheme of the KISS method. (A) Rat heart cytosolic fraction was incubated with glutathione Sepharose beads coated with a GST-tagged kinase to form kinase-substrate complex. After washing, the bound proteins were incubated with ATP/ $\mathrm{Mg}^{2+}$ to occur phosphorylation. These samples were submitted to the LC-MS/MS study. (B) Phosphorylation of kinase interacting proteins. The kinase-substrate complexes of GST-ERK or GST-PKA were incubated with or without ATP to induce phosphorylation. Immunoblot analyses were performed to evaluate the phosphorylation using phospho-T-P and K/R-K/R-X-phospho-S/T antibodies. (C) The effects of inhibitors on the phosphorylation of substrates by the respective kinases. After the formation of the kinase-substrate complexes, they were treated with $100 \mu \mathrm{mol} / \mathrm{L}$ of FR180204 (for ERK) or $50 \mu \mathrm{mol} / \mathrm{L}$ of KT5720 (for PKA), and then incubated in the presence or absence of ATP. Immunoblot analyses were performed to evaluate the phosphorylation using phospho-T-P and K/R-K/R-X-phospho-S/T antibodies. 


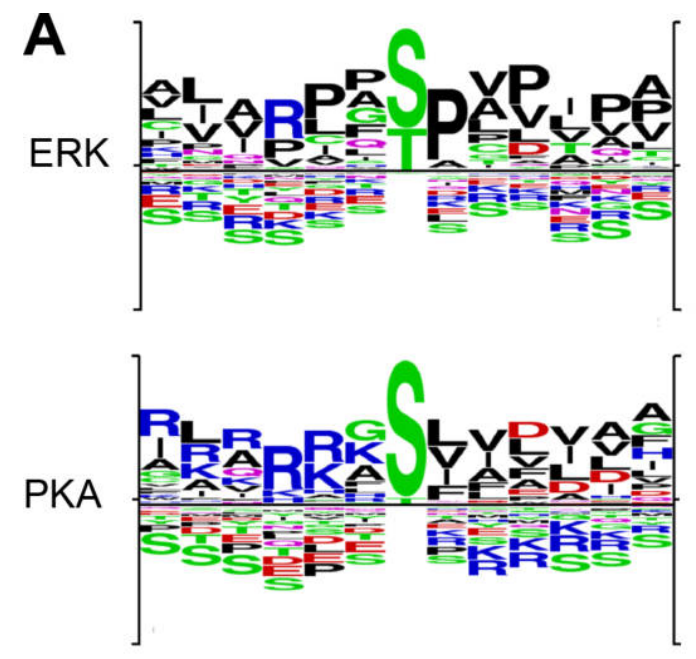

B

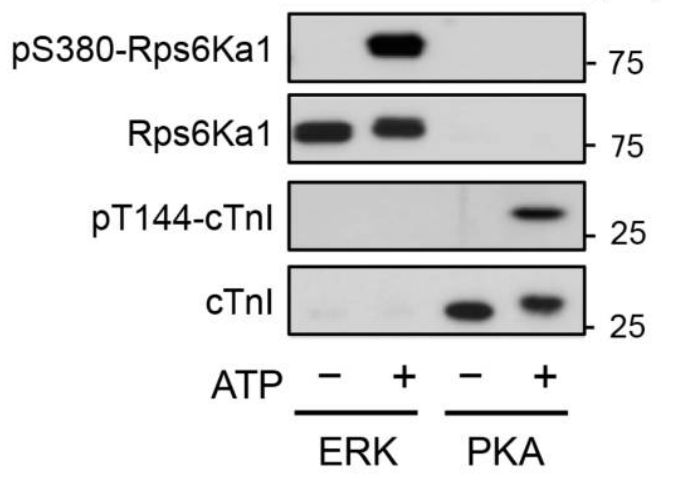

C

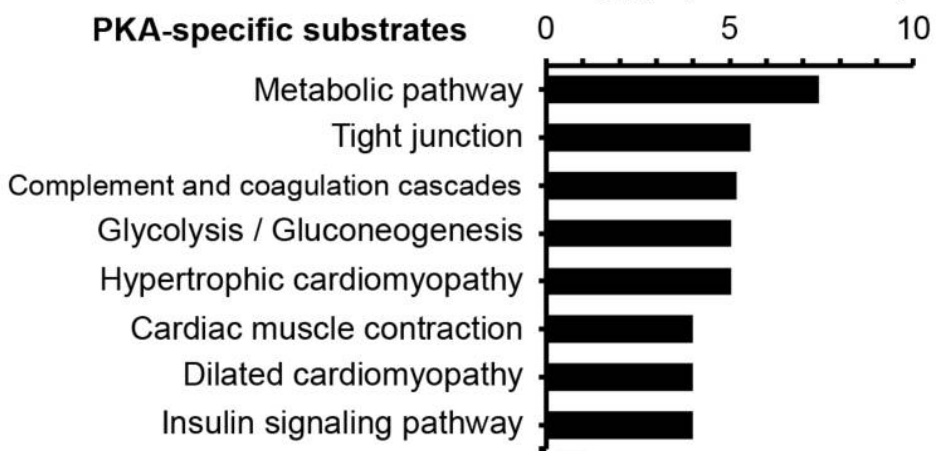

Fig. 2. KISS method efficiently identified the substrate of ERK and PKA in the heart. (A) Sequence alignments of candidate phosphopeptides by Logo Generators. (B) The phosphorylation sites detected in Rps6ka1 and cTnI were confirmed by immunoblot using phosphorylation site-specific. (C) KEGG pathway analysis from phosphoproteins in the KISS method using WEB-based GEne SeT AnaLysis Toolkit. 
A

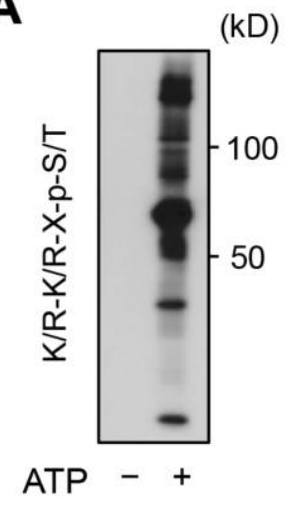

(kD)

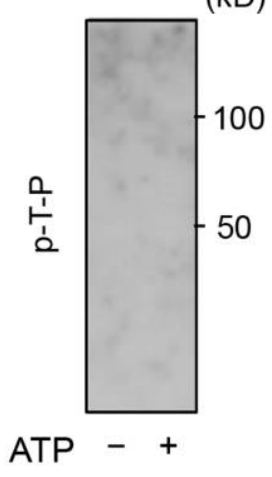

B

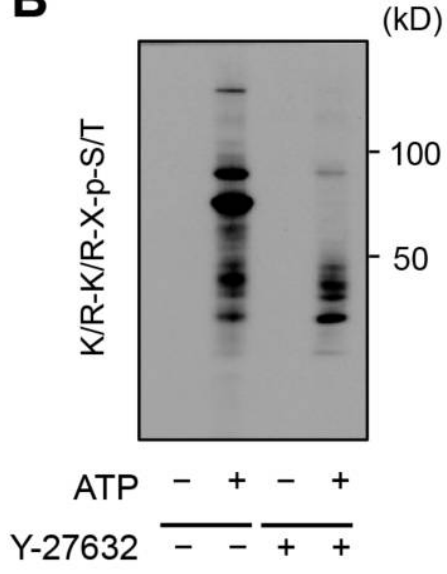

C

\begin{tabular}{|c||c|}
\hline $\begin{array}{c}\text { Cytoskeletal } \\
\text { protein }\end{array}$ & Kinase \\
\hline Add1 & Aak1 \\
Add2 & Adck3 \\
Add3 & Ak4 \\
CARP & Alpk3 \\
Csrp3 & Cdk16 \\
Kif5b & Ckmt2 \\
Lmod2 & Grk5 \\
LOC290704 & Gsk3a \\
LOC312502 & Gsk3b \\
Map1a & Map2k4 \\
Map4 & Mapk7 \\
Mapre3 & Mapkapk2 \\
Mybpc3 & Mylk3 \\
Nrap & Pdk1 \\
Pdlim5 & Pdk4 \\
Sbds & Pfkfb2 \\
Sptbn1 & Pfkm \\
Svil & Prkaca \\
Synpo2 & Prkar2a \\
Tln1 & Prkd1 \\
Tnni3 & Prkd3 \\
Tnnt2 & Slk \\
Tppp & Srpk1 \\
Tppp3 & Stk24 \\
Wipf1 & Stk25 \\
Wipf3 & Taok1 \\
\hline
\end{tabular}

\begin{tabular}{|c|}
\hline GEF/GAP \\
\hline Arhgap12 \\
Arhgef1 \\
Arhgef11 \\
Arhgef12 \\
Arhgef17 \\
Arhgef6 \\
Arhgef7 \\
Rapgef2 \\
\hline
\end{tabular}

Sarcomere protein

CARP

Ckmt2

Csrp3

Fhl2

$\mathrm{Hspb} 1$

LOC290704

Mybpc3

Myom1

Myom2

Pdlim5

Sptbn1

Synpo2

Tnni3

Tnnt2

\begin{tabular}{|c|}
\hline Transcriptional \\
regulator \\
\hline Ankrd17 \\
Btf3 \\
Cand2 \\
CARP \\
Foxk1 \\
Foxo1 \\
Hcfc1 \\
Lpin1 \\
Mecp2 \\
Naca \\
Nkx2-1 \\
Nkx2-4 \\
Nkx2-5 \\
Purb \\
Rbm12 \\
Rbm42 \\
Smyd1 \\
Smyd2 \\
Tdrd3 \\
Tead1 \\
Tead2 \\
Ybx1 \\
Ybx2 \\
Zfr \\
Zhx2 \\
\hline
\end{tabular}

D

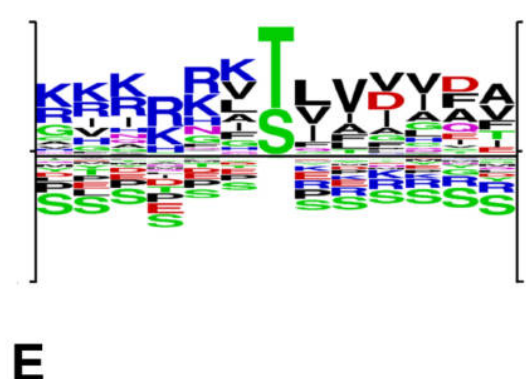

E

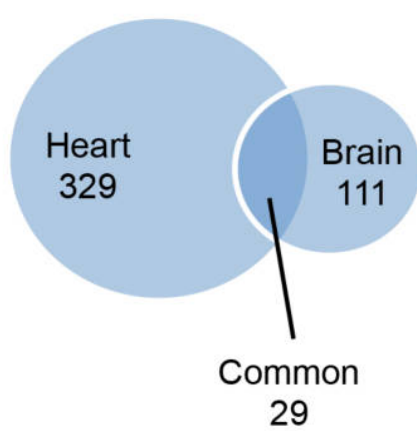

$\mathbf{F}$

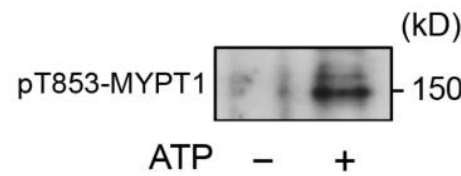

Fig. 3. Substrate screen of Rho-kinase in the heart. (A) Phosphorylation of kinase interacting proteins. The kinase-substrate complexes of GST-Rhokinase-cat were incubates with or without ATP to induce phosphorylation. Immunoblot analyses were performed to evaluate the phosphorylation using antibodies recognizing phospho-T-P and K/R-K/R-X-phospho-S/T. (B) The effects of inhibitor on the phosphorylation of substrates by Rho-kinase. The Rho-kinase-substrate complexes were treated with $100 \mu \mathrm{mol} / \mathrm{L}$ of Y-27632 and then incubated with or without ATP. Immunoblot analyses were performed to evaluate the phosphorylation using K/R-K/R-X-phospho-S/T antibody. (C) Functional classification of the candidate substrates. The candidate substrates were classified into the indicated categories as shown. Cytoskeletal protein, sarcomere protein, kinase, regulators of small GTPases (GEF/GAP), and transcriptional regulator were detected. (D) Sequence alignment of candidate phosphopeptides. (E) Overlapping of Rho-kinase candidate substrates between the heart and brain experiments. (F) The phosphorylation of MYPT1 was confirmed by immunoblot using anti-phospho-specific MYPT1 antibody. 
Tables). Sequence alignment of the predicted phosphorylation sites revealed that Rho-kinase preferentially phosphorylates $(\mathrm{K} / \mathrm{R}) \mathrm{XXp}(\mathrm{S} / \mathrm{T})$ and $(\mathrm{K} / \mathrm{R}) \mathrm{Xp}(\mathrm{S} / \mathrm{T})$ sequences, a finding that is consistent with a previous report (Fig. 3D) (Amano et al., 2010a; Chen et al., 2014). The KISS method showed 329 candidate substrates of Rho-kinase, and only 29 of them overlapped with the substrates obtained from brain, implying the existence of numerous heart specific substrates (Fig. 3E, S4 Table). Rho-kinase-mediated substrates in the heart as well as in the brain included Add1 ( $\alpha$-adducin), Ppp1r12a (MYPT1, MBS), and Scrib, which have been reported as substrates in non-cardiomyocytes. The phosphorylation of myosin phosphatase target subunit 1 (MYPT1) was detected in an ATP-dependent manner by immunoblot, using an anti-phospho-MYPT1 antibody (Fig. $3 F$ ). These results suggest that this method effectively identified the substrates of Rho-kinase whose function in the heart are unknown.

Among the putative Rho-kinase substrates, cardiac ankyrin repeat protein (CARP) is primarily expressed in cardiac muscle and has been described as a transcriptional co-factor that regulates genes involved in the development of cardiac hypertrophy as well as myocardial structural changes, including the expression of the cardiac ventricular-specific myosin light chain-2v (MLC-2v) gene (Mikhailov and Torrado, 2008). Thus, we expected CARP to explain the underlying mechanism of Rho-kinasemediated cardiac diseases, and hereafter focused on CARP. CARP contains a single coiled-coil domain at the Nterminus followed by nuclear localizing signal (NLS), and five conserved ankyrin repeats towards the C-terminus (Fig. 4A). The potential phosphorylation sites detected were T102 and S314 (Fig. 4A). To confirm the screening data, we performed an in vitro phosphorylation assay. GSTtagged CARP-FL was produced in E. coli, and purified protein and Rho-kinase-cat domain were mixed to induce phosphorylation. As a result, Rho-kinase efficiently phosphorylated CARP in a time- and dose-dependent manner (Fig. 4B). We transfected pEF-BOS-GST-CARP-FL (1-319 aa), pEF-BOS-GST-CARP-N-term (1-122 aa), pEF-BOSGST-CARP-C-term (120-319 aa), and their Ala mutants (CARP-FL-AA, CARP-N-102A, CARP-C-314A) into COS7 cells, and the precipitated proteins with glutathione beads were subjected to in vitro phosphorylation assays. Rho-kinase phosphorylated CARP-FL, and CARP-FL-AA mutation resulted in significant reduction in phosphorylation (Fig. 4C, D). Also, Rho-kinase phosphorylated both CARP-N and CARP-C. The CARP-N-102A and CARPC-314A mutations resulted in significant reduction in phosphorylation (Fig. 4E, F). Consistent with the KISS analysis, these results suggest that both T102 and S314 are major phosphorylation sites of Rho-kinase.

\section{CARP binds to 14-3-3C/YWHAZ in a phosphorylation- dependent manner}

To understand the significance of CARP phosphorylation by Rho-kinase, we surveyed the binding proteins of CARP in a phosphorylation-dependent manner. The rat heart cytosol fraction was incubated with glutathione-Sepharose beads coated with phosphorylated or non-phosphorylated GST-CARP by Rho-kinase, and the binding proteins were analysed by mass spectrometry. We obtained 493 candidate binding partners for both samples (S6 Table) and compared these data by dividing the intensity obtained in the phospho group with that in the non-phospho group (Fig. 5A). Among the interacting proteins with 10-fold higher intensities for phosphorylated CARP than the nonphosphorylated form, three species of 14-3-3 proteins $(\varepsilon, \zeta, \gamma)$ exhibited a striking preference for phosphorylated CARP. The 14-3-3 family of proteins are known to bind to the phosphoserine/-threonine-containing motifs and to modulate the function and/or localization of targets in a phosphorylation state-dependent manner (Darling et al., 2005). To confirm the interaction between CARP and 14-3-3, GFP-CARP-WT and -CARP-AA mutant were expressed in COS7 cells, and the cell lysates were incubated with GST-14-3-3 $\zeta$-coated beads. GFP-CARP-WT was co-precipitated with GST-14-3-3ל, which was decreased when the cells were treated with Y-27632, a specific inhibitor of Rho-kinase. On the other hand, GFP-CARP-FL-AA barely bound to 14-3-3 $\zeta$ under the same condition (Fig. 5B, C). Co-

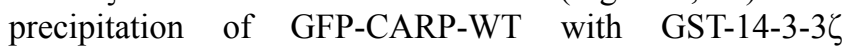
increased under the expression of RhoA constitutive active form (RhoA L63) and decreased under the expression of RhoA dominant-negative form (RhoA N19) (Fig. 5D, E). These results suggest that Rho-kinase phosphorylates CARP at T102 and/or S314 downstream of RhoA in COS7 cells and induces the association with 14-3-3

\section{4-3-3 promotes cytosolic retention of CARP in $\mathrm{H} 9 \mathrm{c} 2$ and $\operatorname{COS} 7$ cells}

CARP has been shown to shuttle between the sarcomere and the nucleus in cardiomyocytes (Mikhailov and Torrado, 2008). We hypothesized that the binding of 14-3-3 to CARP changes its subcellular localization. To assess this hypothesis, GFP-tagged $14-3-3 \zeta$ was transfected to H9c2 cells followed by immunostaining with anti-CARP and anti-GFP antibodies. H9c2 cells are derived from rat embryonic myocardium and express endogenous CARP (Moulik et al., 2009), and have been proposed to have some similarity with neonatal rat cardiomyocyte (Watkins et al., 2011). In cells expressing GFP, endogenous CARP was mainly distributed to the nucleus of $\mathrm{H} 9 \mathrm{c} 2$ cells, similar to untransfected cells. Overexpression of 14-3-3 $\zeta$ increased the cell population with cytoplasmic localization of CARP. However, the phospho motif binding-deficient mutant 
A

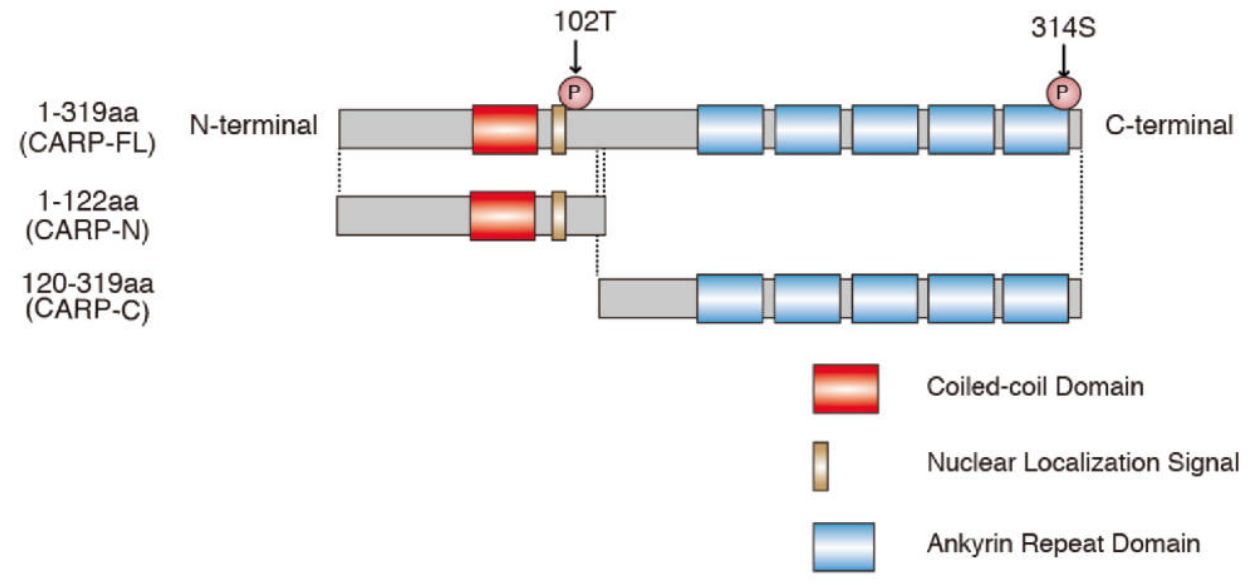

B

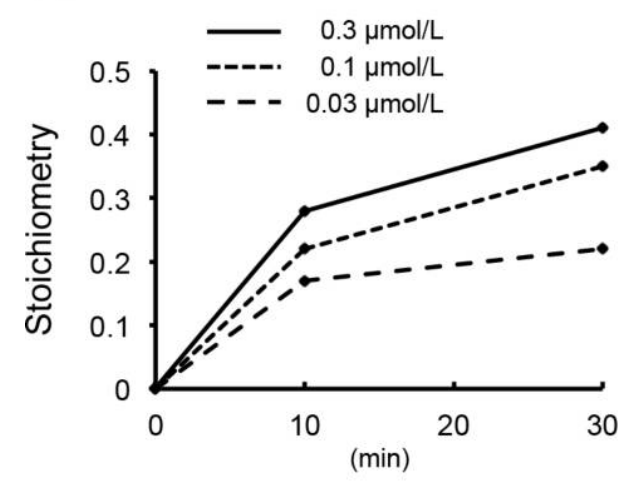

C

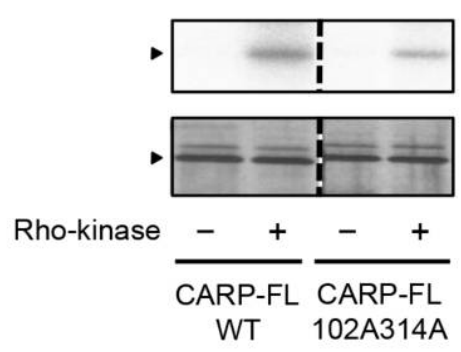

D

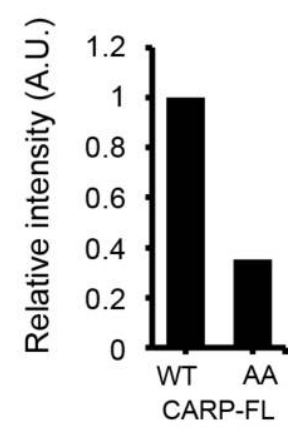

E

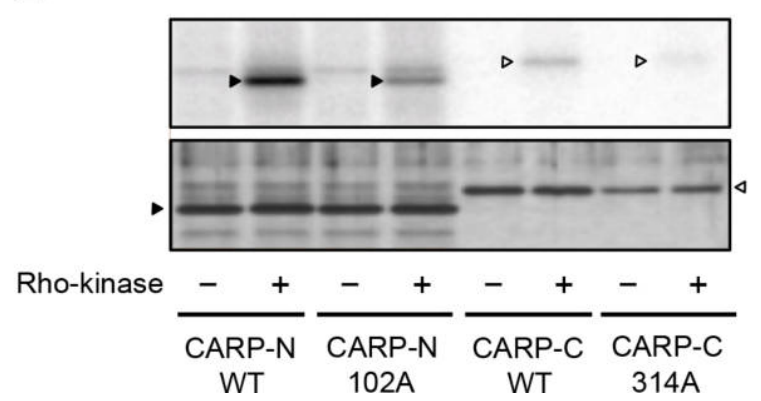

$\mathbf{F}$

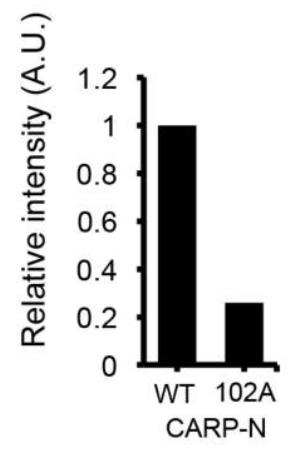

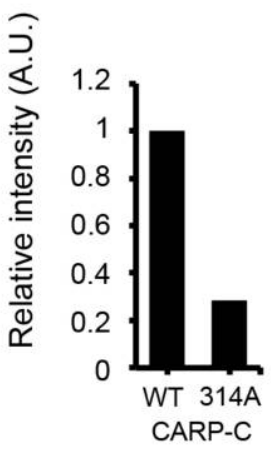

Fig. 4. Rho-kinase phosphorylates CARP at T102 and S314. (A) The domain structure of CARP and deletion constructs used in the experiments. CARP protein contains a single coiled-coil domain, nuclear localizing signal, and five conserved ankyrin repeats. The phosphorylation sites identified by the KISS approach are shown. (B) Phosphorylation of CARP by Rho-kinase in vitro. GST-CARP-FL was incubated with the indicated concentrations of GSTRho-kinase-cat for 10 or $30 \mathrm{~min}$ in the presence of $\left[\gamma^{-32} \mathrm{P}\right]$ ATP. (C, D) Phosphorylation of CARP-FL and its A mutants by Rho-kinase in vitro. GST-CARPFL and GST-CARP-FL-102A314A mutant purified from COS7 cells were phosphorylated using radiolabeled ATP with or without GST-Rho-kinase-cat. Silver staining (bottom). Autoradiography (top). Arrowheads indicate the GST-CARP-FL. (E, F) Phosphorylation of CARP-N, -C and their A mutants by Rho-kinase in vitro. GST-CARP-N, -CARP-C and their A mutants purified from COS7 cells were phosphorylated using radiolabeled ATP with or without GST-Rho-kinase-cat. Silver staining (bottom). Autoradiography (top). Filled arrowheads indicate the GST-CARP-N, and open arrowheads indicate GSTCARP-C. 

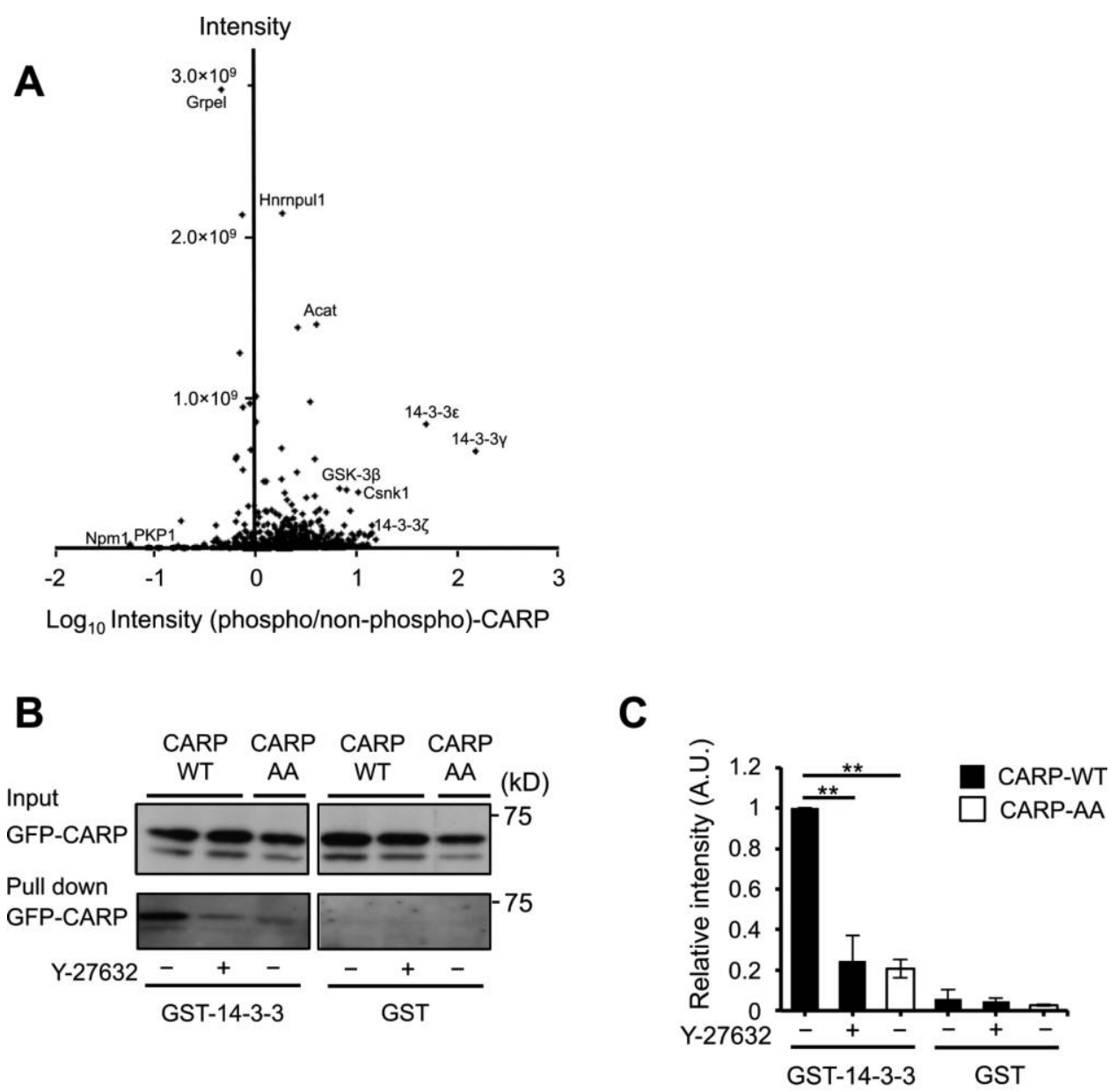

D

$\mathbf{E}$
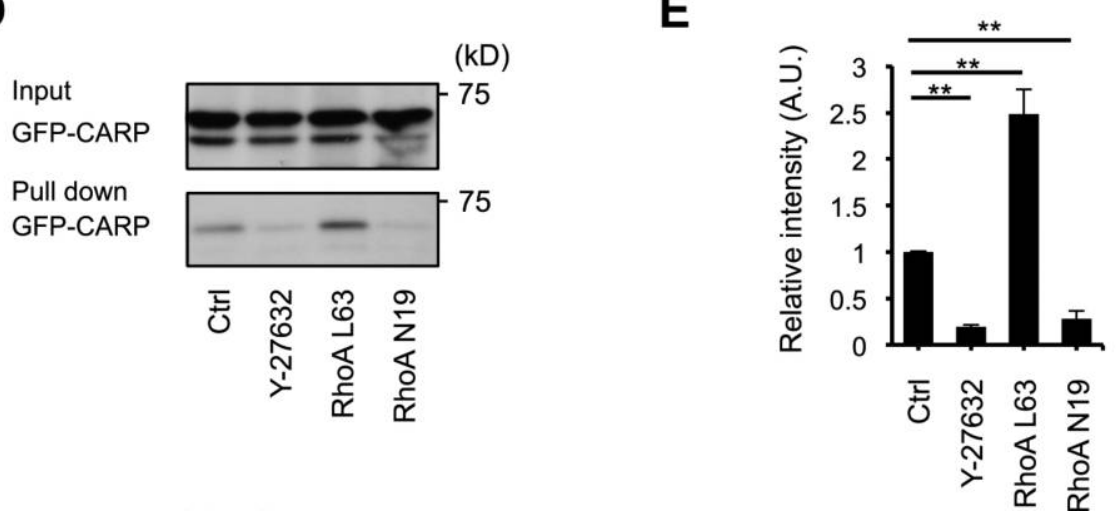

Fig. 5. CARP binds to $14-3-3 \zeta$ in a phosphorylation-dependent manner. (A) Screening of phosphorylated CARP binding partners. The rat heart cytosol fraction was incubated with glutathione-Sepharose beads coated with phosphorylated or non-phosphorylated GST-CARP by Rho-kinase, and the binding proteins were analysed by mass spectrometry. The ratio distributions against the intensities obtained in phospho-CARP samples are shown. These data are presented as the $\log 10$ of the intensity ratios obtained between the phosphorylated and non-phosphorylated samples. (B, C) Rho-kinase-dependent interaction between CARP and 14-3-3 $\zeta$ in COS7 cells. COS7 cells were transfected with GFP-CARP-WT or -AA, and the cells were treated with either DMSO or Y-27632 $(20 \mu \mathrm{mol} / \mathrm{L}, 30 \mathrm{~min})$. (D, E) RhoA constitutive active form (RhoA L63) or dominant negative form (RhoA N19) was co-transfected

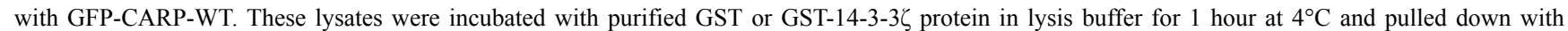
glutathione Sepharose. Pulled down samples were subjected to immunoblot using anti-GFP antibody. These results are representative of three independent experiments. ${ }^{* *}$ indicates a statistically significant difference compared to control at $\mathrm{p}<0.01$. Error bars indicate \pm SEM. 
A

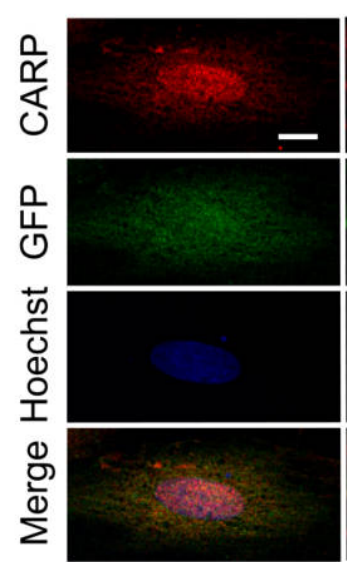

GFP
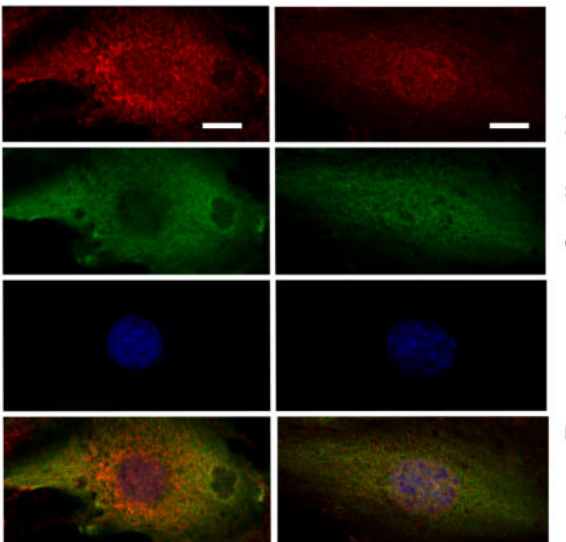

GFP-14-3-3 WT

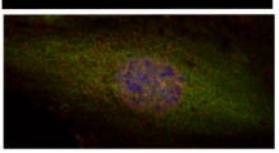

GFP-14-3-3 mutant
B

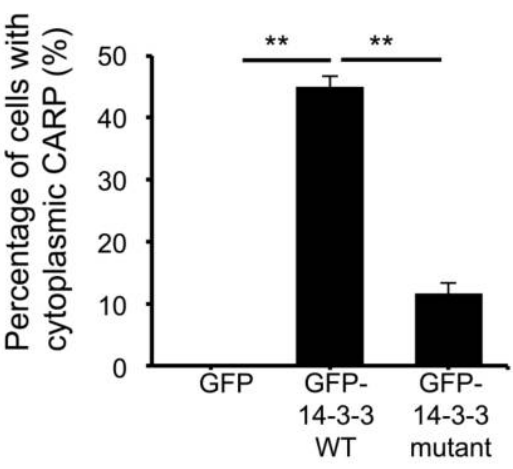

\section{C}
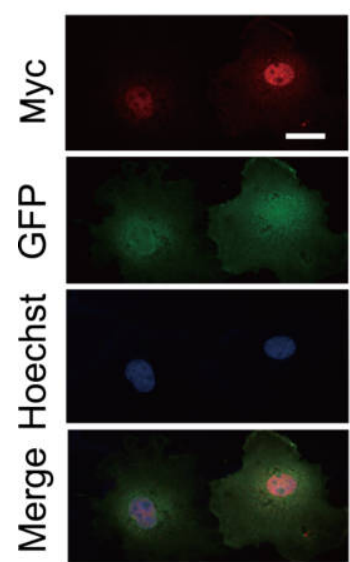

GFP Myc-CARP-WT
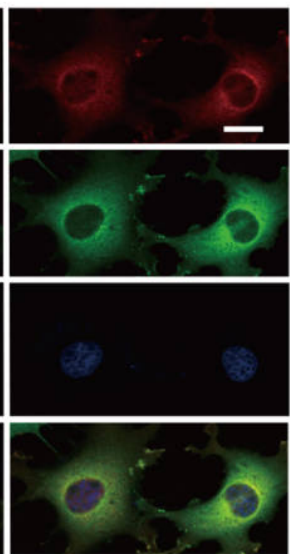

GFP-14-3-3 Myc-CARP-WT
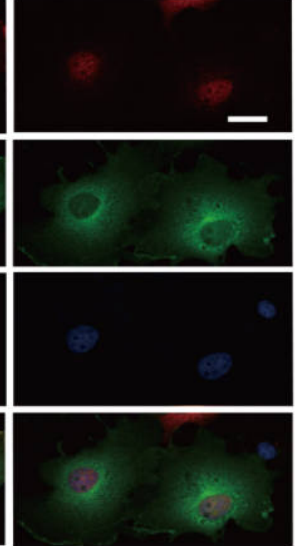

GFP-14-3-3 Myc-CARP-AA
D

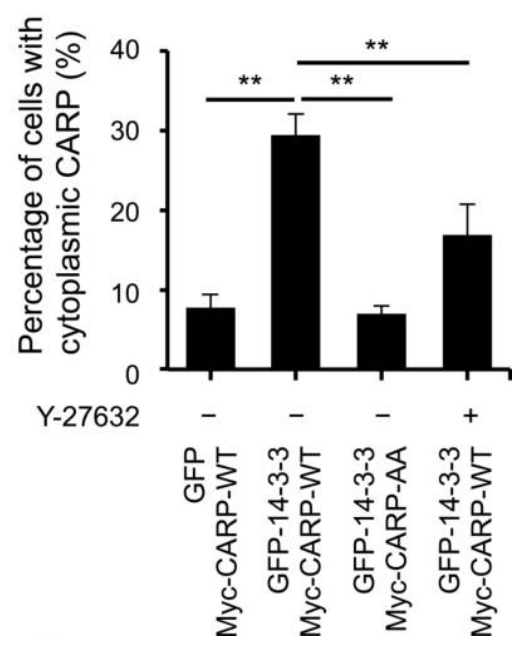

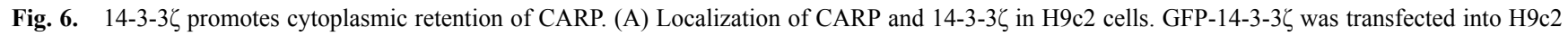
cells followed by immunostaining with anti-CARP and anti-GFP antibodies. Representative images of H9c2 cells transfected with GFP-tagged proteins are

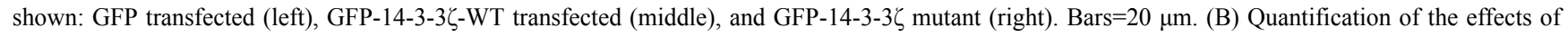

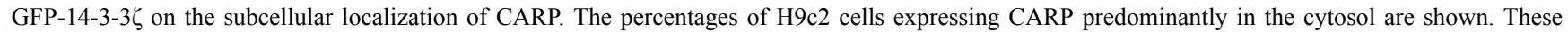

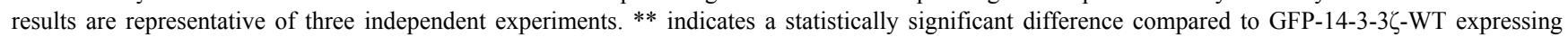

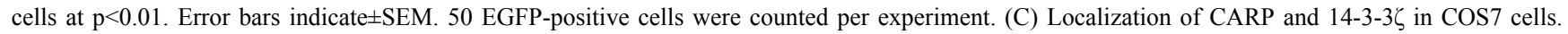
Myc-CARP WT or AA mutant was transfected into COS7 cells in combination with either GFP or GFP-14-3-3 with anti-GFP and anti-myc antibodies. Representative images of COS7 cells co-transfected with Myc-CARP and GFP-tagged protein are shown: GFP/

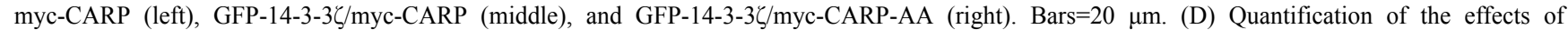

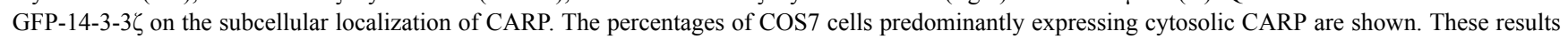
are representative of three independent experiments. ${ }^{*}$ indicates a statistically significant difference compared to control cells at $\mathrm{p}<0.01$. Error bars indicate \pm SEM. At least 50 EGFP-positive cells were counted per experiment. 
A

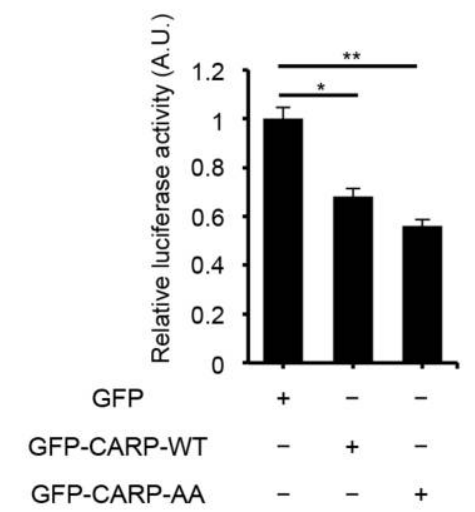

B

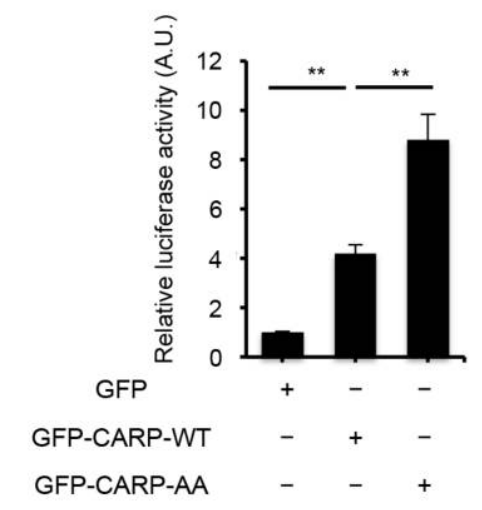

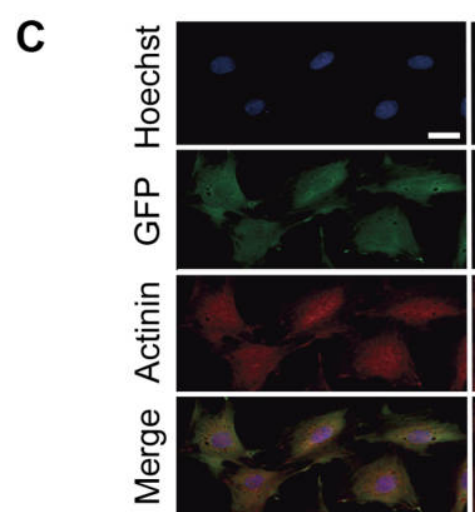

GFP

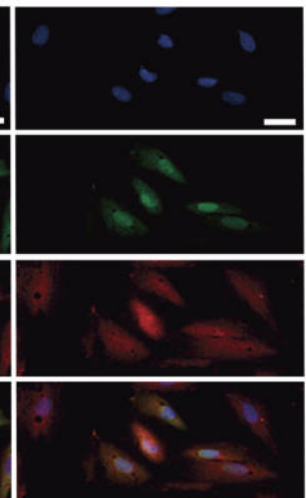

GFP-CARP-WT

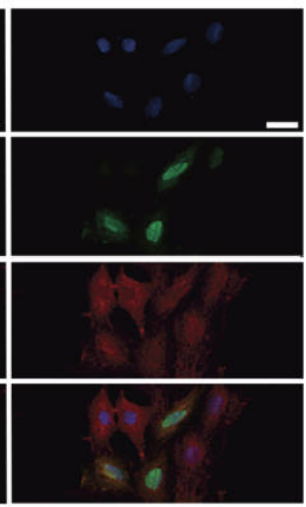

GFP-CARP-AA

D

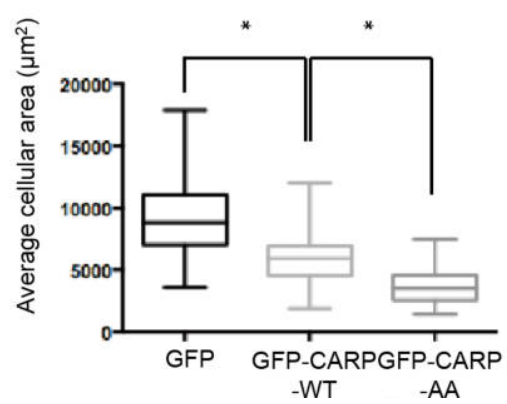

Fig. 7. CARP regulates the cardiac cellular functions depending on its phosphorylation state. (A) Transcriptional activities of the MLC-2v promoter. Neonatal rat cardiomyocytes were grown for $24 \mathrm{~h}$ in 24 -well dishes and then transiently co-transfected with reporter vectors $\left(\mathrm{pGL}_{3}-(\mathrm{MLC}-2 \mathrm{v})_{4} \mathrm{promoter}\right.$ with pRL-TK promoter) and expression vectors for GFP-CARP-WT and -CARP-AA. The cells were lysed in Passive Lysis Buffer $24 \mathrm{~h}$ after transfection, and luciferase activity was measured using the Dual-Luciferase Assay System and a luminometer. Firefly luciferase activities were normalized to their corresponding renilla luciferase activities to correct for the variation in transfection efficiency. The plotted results are representative of three independent experiments performed in sextuplicate. (* indicates a difference from the value of control cells at $\mathrm{p}<0.05$, and $* *$ indicates a difference at $\mathrm{p}<0.01$. Error bars indicate \pm SEM.) (B) Transcriptional activities of p53 responsive promoter. HEK293 cells were grown for $24 \mathrm{~h}$ in 24 -well dishes and then transiently co-transfected with reporter vectors ( $\mathrm{pGL}_{4}$-p53-RE promoter with $\mathrm{pRL}-\mathrm{TK}$ promoter) and expression vectors for GFP-CARP-WT or -CARP-AA. The cells were lysed in Passive Lysis Buffer $24 \mathrm{~h}$ after transfection, and luciferase activity was measured using the Dual-Luciferase Assay System and a luminometer. Firefly luciferase activities were normalized to their corresponding renilla luciferase activities to correct for variation in transfection efficiency. The plotted results are representative of three independent experiments performed in triplicate. (** indicates a difference at $\mathrm{p}<0.01$. Error bars indicate \pm SEM.) (C) Effect of overexpression of CARP-WT and -AA in H9c2 cells. GFP, GFP-CARP-WT, or -AA was transfected into H9c2 cells followed by immunostaining with anti-GFP and anti- $\alpha$-actinin antibodies. Representative images of H9c2 cells transfected with GFP-tagged proteins are shown: GFP transfected (left), GFP-CARP-WT transfected (middle), and GFP-CARP-AA (right). Bars=50 $\mu \mathrm{m}$. (D) Quantification of the effects of GFPCARP on the cell size of $\mathrm{H} 9 \mathrm{c} 2$ cells. The cell area of $\mathrm{H} 9 \mathrm{c} 2$ cells is shown. These results are representative of three independent experiments. $(*$ indicates a difference at $\mathrm{p}<0.05$. Error bars indicate \pm SEM. 100 EGFP-positive cells were counted per experiment.) 
14-3-3 $\zeta$ failed to delocalize CARP (Fig. 6A, B). Next, we assessed whether the expression of 14-3-3 proteins has a role in the localization of exogenously expressed CARPWT and its Ala mutant in COS7 cells, which do not express endogenous CARP. Myc-tagged CARP WT or AA mutant was transfected into COS7 cells in combination with either GFP or GFP-14-3-3 , followed by immunostaining with anti-GFP and anti-myc antibodies. Consistent with H9c2 cells, myc-CARP was principally distributed to the nucleus in cells expressing GFP. Conversely, overexpression of GFP-14-3-3 $\zeta$ delocalized CARP from the nucleus to the cytoplasm, whereas 14-3-3 $\zeta$ failed to delocalize the CARPAA mutant. Inhibition of endogenous Rho-kinase activity by Y-27632 cancelled the effect of $14-3-3 \zeta$ on the localization of CARP (Fig. 6C, D). These results suggest that 14-3-3 contributes to the cytoplasmic retention of CARP.

\section{CARP regulates cardiac cellular functions depending on its phosphorylation state}

CARP has been proposed to regulate the promoter activities of genes re-expressed during cardiac hypertrophy (Mikhailov and Torrado, 2008). CARP has been described not only as a negative regulator of MLC-2v promoter activity (Zou et al., 1997) but also as a positive regulator of $\mathrm{p} 53$ responsive promoter activity (Kojic et al., 2010). Thus, we speculated that CARP changes the promoter activity depending on its phosphorylation state. To test this hypothesis, we performed luciferase assays using the MLC-2v promoter and the p53 responsive promoter. CARP repressed MLC-2v promoter activity in neonatal rat cardiomyocytes and promoted p53 responsive promoter activity in HEK 293 cells, consistent with previous reports (Zou et al., 1997; Kojic et al., 2010). The non-phosphorylatable form of CARP had a stronger effect than the WT form (Fig. $7 \mathrm{~A}, \mathrm{~B})$. These results suggest that these promoter activities are regulated by the phosphorylation state of CARP. As CARP was reported to attenuate cardiac hypertrophy in culture primary cardiac cells (Song et al., 2012), we next examined the effect of CARP on the cell size of H9c2 cardiomyoblast cells. The overexpression of CARP-WT decreased cell size of $\mathrm{H} 9 \mathrm{c} 2$ cells cultured in the $10 \%$ FBScontaining medium, and non-phosphorylatable form of CARP had a stronger effect than that of the WT form (Fig. $7 \mathrm{C}, \mathrm{D})$. These results suggest that cell size of $\mathrm{H} 9 \mathrm{c} 2$ cells is partially regulated by the phosphorylation state of CARP.

\section{Discussion}

We searched for novel substrates of ERK, PKA, and Rhokinase in the heart by combining LC-MS/MS and affinity chromatography. When kinase phosphorylates its substrate, ATP and substrate bind to kinase at the active center and form a substrate-kinase complex (Ubersax and Ferrell,
2007). In our method, we used affinity beads coated with kinases to obtain the kinase substrates. We obtained 382 PKA substrates including 42 known substrates (Table I, S1 Table). The role of PKA to regulate cardiac muscle contraction is relatively well known (Bers, 2002). In this study, we detected several proteins related to cardiac muscle contraction such as cTnI, Mybpc3, Tnnt2, Tpm1, and Myh7. Although cTnI and Mybpc3 has been reported as substrates of PKA, there is no report about the phosphorylation of the other proteins by PKA. It is possible that PKA also phosphorylates the substrates newly identified by this approach in addition to cTnI and Mybpc3 to regulate cardiac contractility cooperatively. PKA-mediated substrate includes proteins related to not only cardiac muscle contraction but also metabolism (Fig. 2C). For example, we detected candidate PKA substrates related to insulin signaling pathway such as Acaca, Gsk3b, Hk1, Phka1, Prkab1, and Pygm in the heart. There are several reports that suggest the relationship between PKA and insulin signaling in the heart (Mangmool et al., 2015); however how PKA influence insulin signaling is little known. The phosphorylation of these proteins might account for the underlying mechanism. Further study will enables us to know the novel function of PKA in the heart. Thus, the KISS method provides us with useful information to suggest novel function of kinases.

In the KISS method, the phosphorylation of substrates was confirmed by the immunoblot using corresponding phospho-motif antibody for each bait kinase (Fig. 1B, Fig. $3 \mathrm{~A}$ ), which was significantly decreased by the specific kinase inhibitors (Fig. 1C, Fig. 3B). These results indicate that the bait kinase substantially phosphorylated the substrates interacted with them under these conditions. Most of the candidate phosphorylation sites revealed by LC-MS/MS analysis matched the phospho-motif sequences of respective kinases, and many known substrates were detected in the ERK and the PKA experiments. Therefore, the KISS method is suitable for the first screen for the physiologically important substrates of target kinase. Nevertheless, some phospho-peptides which did not match the consensus sequence of the bait kinases were detected in these experiments. It is possible that the phosphorylation sites identified by this method include those by other kinases interacted with the bait kinase. Because a few important substrates that do not match the consensus sequences have been reported (Duarte et al., 2014), we cannot exclude the possibility that these sites are really phosphorylated by the bait kinase. Additional assay will be needed to confirm whether these substrates are really phosphorylated by the target kinases.

Recently, Rho-kinase, a major downstream effector of the small GTPase Rho, was shown to be involved in the pathogenesis of cardiac hypertrophy and heart failure through several animal experiments. Cardiac specific deletion of Rho-kinase protects mice from pressure overloadinduced cardiomyocyte hypertrophy through regulation of 


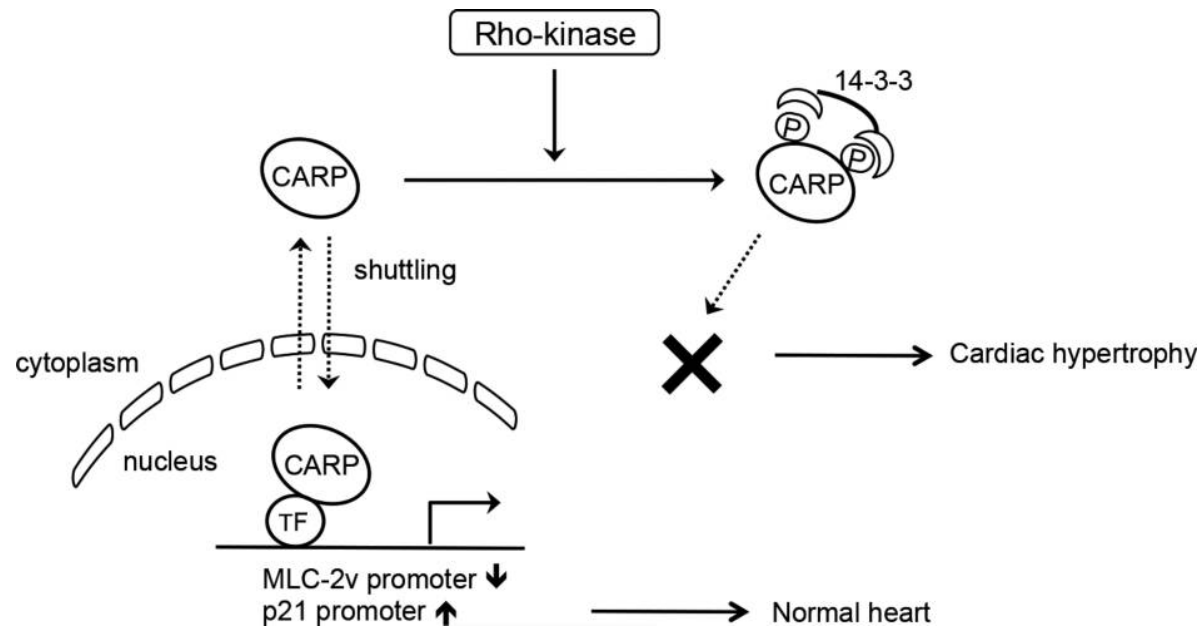

Fig. 8. Schematic diagram showing Rho-kinase/CARP-dependent signalling cascades. The phosphorylation of CARP by Rho-kinase leads to the binding to 14-3-3 and attenuates the nuclear translocation, which would result in an acceleration of the transcriptional activity of genes involved in the development of hypertrophy. Transcription factor (TF).

hypertrophic gene expression, and abnormalities in animal models of heart failure have been ameliorated by long-term treatment with the selective Rho-kinase inhibitor fasudil (Hartmann et al., 2015; Shimokawa and Satoh, 2015). These data suggest that Rho-kinase has an important role in the pathogenesis of these cardiac diseases, most likely through transcriptional regulation. We previously reported that Rho-kinase plays a crucial role in $\mathrm{Ca}^{2+}$-sensitization of smooth muscle contraction. Rho-kinase phosphorylates MYPT1 and inactivates myosin light chain phosphatase, thereby increasing the phosphorylation of MLC followed by smooth muscle contraction (Amano et al., 2010a). For the last 10 years, many substrates of Rho-kinase were studied in neurons, and it was observed that the phosphorylated proteins control nerve axon extension (Namba et al., 2015). In contrast to vascular cells and neuronal cells, less is known about the substrates of Rho-kinase in cardiomyocytes. Although Rho-kinase has been shown to play an important role in the pathogenesis of cardiac hypertrophy and heart failure, its cardiac-specific substrate has not been reported, which urged us to screen its substrates by the KISS method. Originally, the KISS method was developed using rat brain lysates and effectively identified the substrate of several kinases. Compared with the data in the brain experiment, the majority of candidate substrates were detected only in the heart experiment, suggesting the importance of the heart tissue to identify heart-specific kinase substrates. The KISS method facilitates the identification of tissue-specific kinase substrates by comparing the substrates between the multiple organs.

Among the candidate substrates, we confirmed CARP as a novel substrate of Rho-kinase by additional cell biological experiments. CARP has been described as a transcriptional co-factor that regulates genes involved in the development of cardiac hypertrophy as well as myocardial structural changes, and proposed to exert anti-hypertrophic effects (Mikhailov and Torrado, 2008). Several CARP mutations are found in patients with cardiomyopathy, and these abnormalities are supposed to be involved in the pathogenesis of both hypertrophic and dilated cardiomyopathy (Arimura et al., 2009; Crocini et al., 2013; Moulik et $a l ., 2009)$. Thus, we expected CARP to explain the underlying mechanism of Rho-kinase-mediated cardiac disease. Rho-kinase phosphorylated CARP at T102 and S314. Phosphorylation of CARP by Rho-kinase induced its binding to 14-3-3. CARP was recruited from the nucleus to the cytoplasm under the overexpression of exogenous $14-3-3 \zeta$, and the T102A/S314A mutant of CARP that weakly binds to

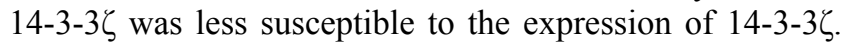
CARP has a putative nuclear localization signal (NLS) at residues 94-98, and we found that deletion of this region results in the cytoplasmic localization of CARP in cultured cells (unpublished observation), suggesting that the nuclear localization of CARP is chiefly dependent on the NLS sequence. The phosphorylation site T102 is close to a putative NLS, and it is likely that 14-3-3 protein binding masks the function of NLS and influences the intracellular localization of CARP. We also demonstrated that the T102A/ S314A mutant had more prominent effects on MLC-2v, a cardiac myosin molecule, and p53 responsive promoters than WT as a transcriptional regulator, which is in accordance with the results of 14-3-3-binding and localization of CARP. Up-regulation of MLC-2 $v$ has been used extensively as a marker protein for myocardial hypertrophy as well as directly causes cardiac hypertrophy (Lee et al., 1988). Conversely, cyclin-dependent kinase inhibitor p21, whose expression is tightly controlled by $\mathrm{p} 53$ responsive promoter, is recognized as an anti-hypertrophic factor (Frey 
and Olson, 2003). These results are consistent with the observation that T102A/S314A mutant of CARP decreased cell size of $\mathrm{H} 9 \mathrm{c} 2$ cells more efficiently than CARP-WT. Taken together, these results suggest that the phosphorylation of CARP by Rho-kinase leads to the binding to 14-3-3 and attenuates the nuclear translocation, which would result in an acceleration of the transcriptional activity of genes involved in the development of hypertrophy (Fig. 8). Further study will be needed to fully understand the meaning and the way of CARP phosphorylation in the heart.

As most studies on Rho-kinase have been focused on the actin cytoskeleton and little is known about the role in regulating gene and protein expression, these results broaden the range of Rho-kinase functions rather than previously reported. Many substrates still require study to completely know the function of Rho-kinase in the heart. Further study will enable us to know more clearly the function of Rhokinase in the heart.

\section{Conclusion}

We performed an exhaustive substrate screening of ERK, PKA, and Rho-kinase in the heart and detected a number of potential substrates. Among them, CARP was confirmed as a novel substrate of Rho-kinase. The KISS method can serve as a good initial screening strategy for exhaustive screening of substrate kinase in the heart, and could provide useful information that may help to elucidate cardiac functions and diseases.

Acknowledgments. We thank Dr. E. Villard (Universite Pierre et Marie Curie Paris 6, IFR14, UMR-S956, Paris, France) for providing the luciferase vector, and Drs. Y. Kirii and K. Yoshino (Carna Biosciences, Kobe, Japan) for providing GST-PKA and GST-MAPK1, Dr. F. Ishidate for help with acquiring and analyzing images, Y. Kanazawa for technical assistance, all members of the Kaibuchi lab for discussions and technical support, and T. Ishii for secretarial assistance. We acknowledge the Division of Medical Research Engineering of the Nagoya University Graduate School of Medicine for the use of luminometer (Power Scan4, DS Pharma Biomedical), the Radioisotope Center at the Medical Branch of the Nagoya University School of Medicine (N. Hamada and Y. Nakamura). This study was supported by Grant-in-Aid for Scientific Research (S-20227006, A-25251021, C-23590357, and C-26461127) from the Ministry of Education, Culture, Sports, Science and Technology of Japan (MEXT), and the Hori Science Promotion Foundation.

\section{References}

Amano, M., Mukai, H., Ono, Y., Chihara, K., Matsui, T., Hamajima, Y., Okawa, K., Iwamatsu, A., and Kaibuchi, K. 1996. Identification of a putative target for Rho as the serine-threonine kinase protein kinase N. Science (New York, NY), 271: 648-650.

Amano, M., Chihara, K., Nakamura, N., Kaneko, T., Matsuura, Y., and Kaibuchi, K. 1999. The $\mathrm{COOH}$ terminus of Rho-kinase negatively regulates rho-kinase activity. J. Biol. Chem., 274: 32418-32424.

Amano, M., Nakayama, M., and Kaibuchi, K. 2010a. Rho-kinase/ROCK: A key regulator of the cytoskeleton and cell polarity. Cytoskeleton (Hoboken, NJ), 67: 545-554.
Amano, M., Tsumura, Y., Taki, K., Harada, H., Mori, K., Nishioka, T., Kato, K., Suzuki, T., Nishioka, Y., Iwamatsu, A., and Kaibuchi, K. 2010b. A proteomic approach for comprehensively screening substrates of protein kinases such as Rho-kinase. PLoS ONE, 5: e8704.

Amano, M., Hamaguchi, T., Shohag, M.H., Kozawa, K., Kato, K., Zhang, X., Yura, Y., Matsuura, Y., Kataoka, C., Nishioka, T., and Kaibuchi, K. 2015. Kinase-interacting substrate screening is a novel method to identify kinase substrates. J. Cell Biol., 209: 895-912.

Arimura, T., Bos, J.M., Sato, A., Kubo, T., Okamoto, H., Nishi, H., Harada, H., Koga, Y., Moulik, M., Doi, Y.L., Towbin, J.A., Ackerman, M.J., and Kimura, A. 2009. Cardiac ankyrin repeat protein gene (ANKRD1) mutations in hypertrophic cardiomyopathy. Journal of the American College of Cardiology, 54: 334-342.

Bers, D.M. 2002. Cardiac excitation-contraction coupling. Nature, 415: $198-205$

Chen, C., Ha, B.H., Thevenin, A.F., Lou, H.J., Zhang, R., Yip, K.Y., Peterson, J.R., Gerstein, M., Kim, P.M., Filippakopoulos, P., Knapp, S., Boggon, T.J., and Turk, B.E. 2014. Identification of a major determinant for serine-threonine kinase phosphoacceptor specificity. Mol. Cell, 53: 140-147.

Cox, J. and Mann, M. 2008. MaxQuant enables high peptide identification rates, individualized p.p.b.-range mass accuracies and proteomewide protein quantification. Nat. Biotechnol., 26: 1367-1372.

Crocini, C., Arimura, T., Reischmann, S., Eder, A., Braren, I., Hansen, A., Eschenhagen, T., Kimura, A., and Carrier, L. 2013. Impact of ANKRD1 mutations associated with hypertrophic cardiomyopathy on contraction parameters of engineered heart tissue. Basic Res. Cardiol., 108: 349 .

Darling, D.L., Yingling, J., and Wynshaw-Boris, A. 2005. Role of 14-3-3 proteins in eukaryotic signaling and development. Curr. Top. Dev. Biol., 68: $281-315$.

Duarte, M.L., Pena, D.A., Nunes Ferraz, F.A., Berti, D.A., Paschoal Sobreira, T.J., Costa-Junior, H.M., Abdel Baqui, M.M., Disatnik, M.H., Xavier-Neto, J., Lopes de Oliveira, P.S., and Schechtman, D. 2014. Protein folding creates structure-based, noncontiguous consensus phosphorylation motifs recognized by kinases. Sci. Signal., 7: ra105.

Duboscq-Bidot, L., Charron, P., Ruppert, V., Fauchier, L., Richter, A., Tavazzi, L., Arbustini, E., Wichter, T., Maisch, B., Komajda, M., Isnard, R., and Villard, E. 2009. Mutations in the ANKRD1 gene encoding CARP are responsible for human dilated cardiomyopathy. Eur. Heart J., 30: $2128-2136$.

Frey, N. and Olson, E.N. 2003. Cardiac hypertrophy: the good, the bad, and the ugly. Annu. Rev. Physiol., 65: 45-79.

Hartmann, S., Ridley, A.J., and Lutz, S. 2015. The Function of RhoAssociated Kinases ROCK1 and ROCK2 in the Pathogenesis of Cardiovascular Disease. Frontiers in pharmacology, 6: 276.

Huttlin, E.L., Jedrychowski, M.P., Elias, J.E., Goswami, T., Rad, R., Beausoleil, S.A., Villen, J., Haas, W., Sowa, M.E., and Gygi, S.P. 2010. A tissue-specific atlas of mouse protein phosphorylation and expression. Cell, 143: 1174-1189.

Kato, K., Yazawa, T., Taki, K., Mori, K., Wang, S., Nishioka, T., Hamaguchi, T., Itoh, T., Takenawa, T., Kataoka, C., Matsuura, Y., Amano, M., Murohara, T., and Kaibuchi, K. 2012. The inositol 5phosphatase SHIP2 is an effector of RhoA and is involved in cell polarity and migration. Mol. Biol. Cell, 23: 2593-2604.

Kojic, S., Nestorovic, A., Rakicevic, L., Belgrano, A., Stankovic, M., Divac, A., and Faulkner, G. 2010. A novel role for cardiac ankyrin repeat protein Ankrd1/CARP as a co-activator of the p53 tumor suppressor protein. Arch. Biochem. Biophys., 502: 60-67.

Lee, H.R., Henderson, S.A., Reynolds, R., Dunnmon, P., Yuan, D., and Chien, K.R. 1988. Alpha 1-adrenergic stimulation of cardiac gene transcription in neonatal rat myocardial cells. Effects on myosin light chain-2 gene expression. J. Biol. Chem., 263: 7352-7358. 
Lundby, A., Andersen, M.N., Steffensen, A.B., Horn, H., Kelstrup, C.D., Francavilla, C., Jensen, L.J., Schmitt, N., Thomsen, M.B., and Olsen, J.V. 2013. In vivo phosphoproteomics analysis reveals the cardiac targets of beta-adrenergic receptor signaling. Sci. Signal., 6: rs 11 .

Mangmool, S., Denkaew, T., Phosri, S., Pinthong, D., Parichatikanond, W., Shimauchi, T., and Nishida, M. 2015. Sustained betaAR stimulation mediates cardiac insulin resistance in a PKA-dependent manner. Mol. Endocrinol. (Baltimore, Md), me20151201.

Mikhailov, A.T. and Torrado, M. 2008. The enigmatic role of the ankyrin repeat domain 1 gene in heart development and disease. Int. J. Dev. Biol., 52: 811-821.

Moulik, M., Vatta, M., Witt, S.H., Arola, A.M., Murphy, R.T., McKenna, W.J., Boriek, A.M., Oka, K., Labeit, S., Bowles, N.E., Arimura, T., Kimura, A., and Towbin, J.A. 2009. ANKRD1, the gene encoding cardiac ankyrin repeat protein, is a novel dilated cardiomyopathy gene. Journal of the American College of Cardiology, 54: 325-333.

Namba, T., Funahashi, Y., Nakamuta, S., Xu, C., Takano, T., and Kaibuchi, K. 2015. Extracellular and Intracellular Signaling for Neuronal Polarity. Physiol. Rev., 95: 995-1024.

Nishimura, T., Kato, K., Yamaguchi, T., Fukata, Y., Ohno, S., and Kaibuchi, K. 2004. Role of the PAR-3-KIF3 complex in the establishment of neuronal polarity. Nat. Cell Biol., 6: 328-334.

Shimokawa, H. and Satoh, K. 2015. ATVB Plenary Lecture: translational research on rho-kinase in cardiovascular medicine. Arterioscl. Throm.
Vas. Biol., 35: 1756-1769.

Song, Y., Xu, J., Li, Y., Jia, C., Ma, X., Zhang, L., Xie, X., Zhang, Y., Gao, X., Zhang, Y., and Zhu, D. 2012. Cardiac ankyrin repeat protein attenuates cardiac hypertrophy by inhibition of ERK1/2 and TGF-beta signaling pathways. PLoS ONE, 7: e50436.

Tilley, D.G. 2011. G protein-dependent and G protein-independent signaling pathways and their impact on cardiac function. Circ. Res., 109: 217230.

Ubersax, J.A. and Ferrell, J.E., Jr. 2007. Mechanisms of specificity in protein phosphorylation. Nat. Rev. Mol. Cell Biol., 8: 530-541.

Watkins, S.J., Borthwick, G.M., and Arthur, H.M. 2011. The H9C2 cell line and primary neonatal cardiomyocyte cells show similar hypertrophic responses in vitro. In vitro Cell. Dev.-A., 47: 125-131.

Yan, J.X., Packer, N.H., Gooley, A.A., and Williams, K.L. 1998. Protein phosphorylation: technologies for the identification of phosphoamino acids. J. Chromatogr. A, 808: 23-41.

Zou, Y., Evans, S., Chen, J., Kuo, H.C., Harvey, R.P., and Chien, K.R. 1997. CARP, a cardiac ankyrin repeat protein, is downstream in the Nkx2-5 homeobox gene pathway. Development (Cambridge, England), 124: $793-804$.

(Received for publication, May 23, 2016, accepted, June 10, 2016

and published online, June 23, 2016) 